\title{
Importance of Specimen Type and Quality in Diagnosing Middle East Respiratory Syndrome
}

Hee Jae Huh, M.D. ${ }^{1, *}$, Jae-Hoon Ko, M.D. ${ }^{2, *}$, Young-Eun Kim, M.D. ${ }^{1}$, Chang-Hun Park, M.D. ${ }^{1}$, Geehay Hong, M.D. ${ }^{1}$, Rihwa Choi, M.D. ${ }^{1}$, Shinae Yu, M.D. ${ }^{1}$, Sun Young Cho, M.D. ${ }^{2,4}$, Ji-Man Kang, M.D. ${ }^{3}$, Myoung-Keun Lee, M.T. ${ }^{1}$, Chang-Seok Ki, M.D. ${ }^{1}$, Eun-Suk Kang, M.D. ${ }^{1}$, Nam Yong Lee, M.D. ${ }^{1}$, Jong-Won Kim, M.D. ${ }^{1}$, Yae-Jean Kim, M.D. ${ }^{3,4}$, Young Eun Ha, M.D. ${ }^{2}$, Cheol-In Kang, M.D. ${ }^{2}$, Doo Ryeon Chung, M.D. ${ }^{2}$, Kyong Ran Peck, M.D. ${ }^{2}$, and Jae-Hoon Song, M.D. ${ }^{2}$ Department of Laboratory Medicine and Genetics ${ }^{1}$, Samsung Medical Center, Sungkyunkwan University School of Medicine; Division of Infectious Diseases², Department of Medicine, Samsung Medical Center, Sungkyunkwan University School of Medicine; Department of Pediatrics ${ }^{3}$, Samsung Medical Center, Sungkyunkwan University School of Medicine; Infection Control Office ${ }^{4}$, Samsung Medical Center, Seoul, Korea

\section{Dear Editor,}

From May to July 2015, there was a hospital-associated outbreak in South Korea reporting 186 laboratory-confirmed cases. Highly sensitive and specific laboratory diagnostic tests are important for the control of Middle East respiratory syndrome coronavirus (MERS-CoV) outbreaks. Clinical guidelines for the molecular diagnosis of MERS-CoV are based on the interim recommendations from the WHO, the United States Centers for Disease Control and Prevention (US CDC), and other available resources [1-4]. To date, only a few studies have reported the molecular detection of this disease during an outbreak [5-8]. To validate the current guidelines for MERS-CoV laboratory testing, we retrospectively reviewed the results of MERS-CoV real-time reverse transcription (rRT)-PCR assays in the Korean tertiary care hospital with the largest number of MERS cases (Samsung Medical Center). The Institutional Review Board of the Samsung Medical Center (IRB \#2015-06-201) approved this study.

From June 1 to July 5, 2015, clinical specimens were screened by rRT-PCR testing targeting the upstream E region (upE) using PowerChek MERS real-time PCR kits (Kogene Biotech, Seoul,
Korea) after viral RNA extraction using the QIAamp DSP Viral RNA Mini kit (Qiagen cat\# 61904; Qiagen, Hilden, Germany) or the MagNA Pure 96 system (Roche Applied Science, Manheim, Germany) according to the manufacturer's instructions. Screenpositive specimens were confirmed by the subsequent amplification of ORF1a. A positive test result was defined as a well-defined exponential fluorescence curve that crossed a threshold of $\leq 35$ cycles for both upE and ORFla. For inconclusive results, a specimen was considered "equivocal" if the upE result was positive, but the cycle threshold (Ct) value for ORFla was between 35 and 40 . Additionally, we interpreted the result as "indeterminate" if: 1) the upE result was positive, but the Ct value for ORF1a was undetected or 2) the Ct value for upE was between 35 and $40[3,9]$.

In total, 5,330 MERS-CoV rRT-PCR results from 3,484 individuals were available for analysis, including 4,291 sputums, 145 tracheal aspirates, 732 nasopharyngeal swabs (NPSs), 35 oropharyngeal swabs, 62 nasopharyngeal and oropharyngeal swabs, and 65 other specimens (Table 1). A total of 150 specimens from 51 patients were positive, and 42 yielded inconclu-
Received: May 28, 2016

Revision received: July 10, 2016

Accepted: October 6, 2016

\section{Corresponding author: Chang-Seok $\mathrm{Ki}$}

Department of Laboratory Medicine and Genetics, Samsung Medical Center, Sungkyunkwan University School of Medicine, 81, Irwon-ro, Gangnam-gu, Seoul 06351, Korea

Tel: +82-2-3410-2709, Fax: +82-2-3410-2719, E-mail: changski@skku.edu *These authors contributed equally to this article.

\section{(c) The Korean Society for Laboratory Medicine.}

This is an Open Access article distributed under the terms of the Creative Commons Attribution Non-Commercial License (http://creativecommons.org/licenses/by-nc/4.0) which permits unrestricted non-commercial use, distribution, and reproduction in any medium, provided the original work is properly cited. 
Table 1. Distribution of MERS-CoV rRT-PCR results by specimen type

\begin{tabular}{|c|c|c|c|c|c|}
\hline \multirow{3}{*}{ Type of specimen } & \multicolumn{5}{|c|}{ MERS-CoV rRT-PCR assay results } \\
\hline & \multirow{2}{*}{ Positive } & \multicolumn{2}{|c|}{ Inconclusive } & \multirow{2}{*}{ - Negative } & \multirow{2}{*}{ Total } \\
\hline & & Equivoc & ermi & & \\
\hline URT & 1 & 1 & 2 & 825 & 829 \\
\hline NPS & 1 & 1 & 2 & 728 & 732 \\
\hline OPS & 0 & 0 & 0 & 35 & 35 \\
\hline NP/OP swab & 0 & 0 & 0 & 62 & 62 \\
\hline LRT & 142 & 14 & 19 & 4,261 & 4,436 \\
\hline Sputum & 112 & 11 & 18 & 4,150 & 4,291 \\
\hline Tracheal aspirate & 30 & 3 & 1 & 111 & 145 \\
\hline Others & 7 & 2 & 4 & 52 & 65 \\
\hline Whole blood & 7 & 2 & 2 & 32 & 43 \\
\hline Urine & 0 & 0 & 2 & 16 & 18 \\
\hline Other & 0 & 0 & 0 & 4 & 4 \\
\hline Total & 150 & 17 & 25 & 5,138 & 5,330 \\
\hline
\end{tabular}

Abbreviations: MERS-CoV, Middle East respiratory syndrome coronavirus; $r R T$, real-time reverse transcription; LRT, lower respiratory tract; NPS, nasopharyngeal swab; NP/OP, nasopharyngeal and oropharyngeal; OPS, oropharyngeal swab; URT, upper respiratory tract. sive results. Among the inconclusive results, 17 were equivocal, and 25 were indeterminate. Of the 143 positive respiratory specimens, 142 were lower respiratory tract (LRT) specimens, while only one upper respiratory tract (URT) specimen tested positive for MERS-CoV.

In early phases of the outbreak in the present study, initial diagnostic MERS-CoV rRT-PCR assays were simultaneously conducted by using both sputum and NPS specimens in 36 individuals. From sputum specimens, two, six, and one specimens were positive, equivocal, and indeterminate, respectively. However, only one equivocal result was obtained from NPS specimens. The detection rate for NPS specimens was significantly lower than that for sputum specimens in both upE and ORFla tests $(P=0.0078$ and 0.0156 , respectively). The low virus detection rate in NPS specimens early in MERS-CoV infection illustrates the importance of specimen type for diagnosis. It is suggested that URT specimens are not suitable for diagnosing MERS-CoV infections. On the basis of the WHO interim recommendations, LRT specimens are preferred for detecting MERS-

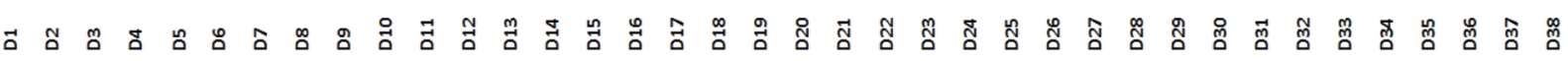

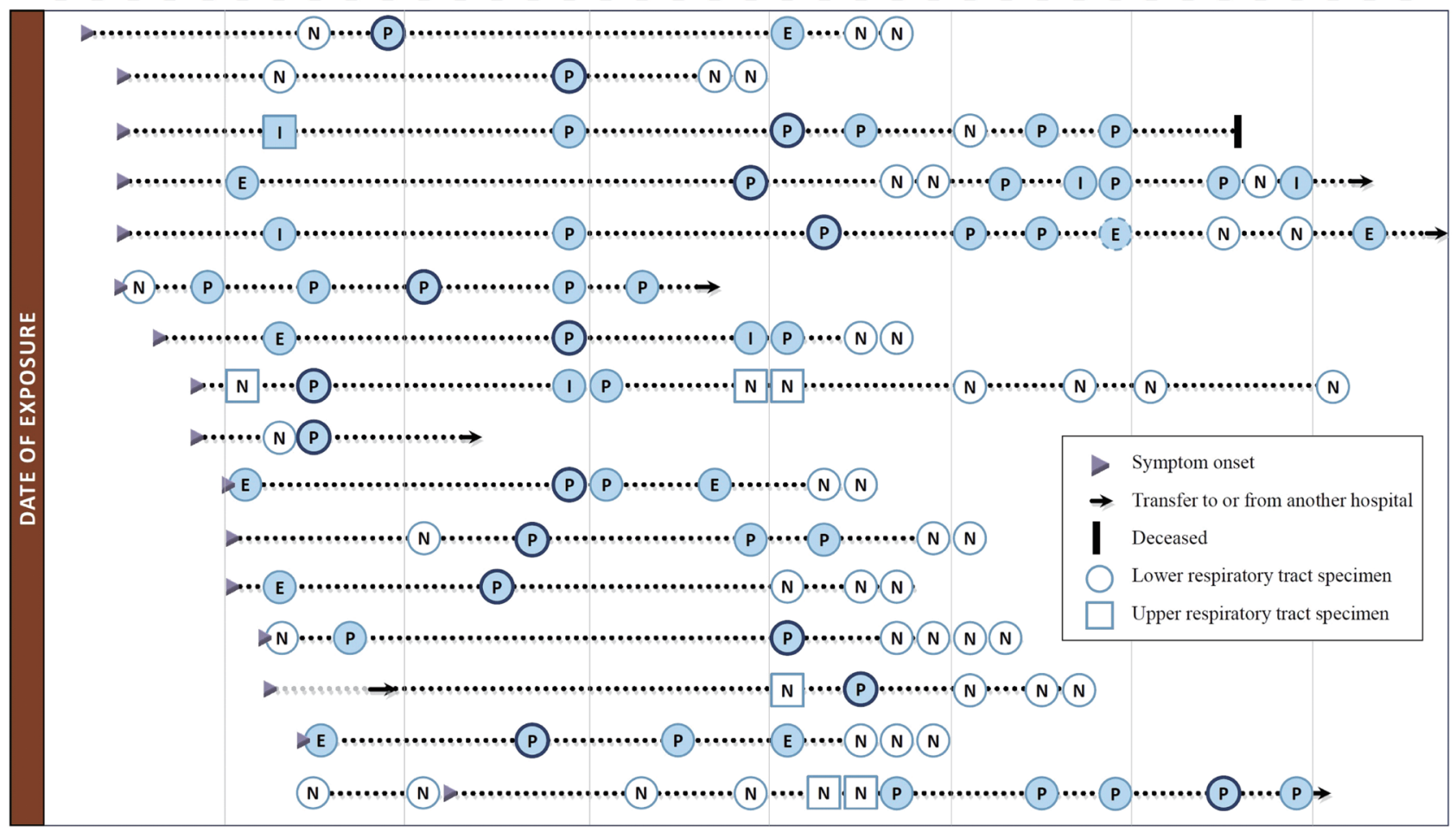

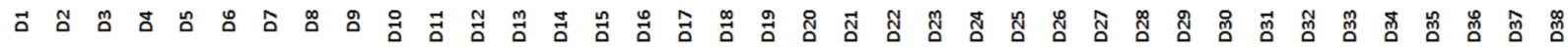

Fig. 1. Schematic representation of virus detection in respiratory tract specimens for 16 confirmed cases with negative or inconclusive results on initial testing. Positive or inconclusive results (closed); negative results (open). A dark ringed circle represents the peak cycle threshold (Ct) value.

Abbreviations: E, equivocal; I, indeterminate; $\mathrm{N}$, negative; $\mathrm{P}$, positive. 
CoV RNA, but collection of URT specimens in addition to LRT is recommended whenever possible [2]. US CDC guidelines endorse the collection of LRT, URT, and serum specimen types for testing [10]. Although simultaneous specimen collection is ideal, it is not feasible because of practical limitations during large and complex outbreaks. On the basis of our results, it seems sufficient to obtain LRT specimens prior to URT or other specimens, especially in early-stage diagnostic testing.

In the study period, 42 inconclusive results were obtained, including 17 equivocal results and 25 indeterminate results. In many of the confirmed MERS patients (16/51, 31.4\%), the initial tests were negative or inconclusive, with not only URT but also LRT specimens. Fig. 1 shows a schematic plot of the detection for MERS-CoV RNA in respiratory tract specimens for the 16 laboratory-confirmed cases. Of note, four consecutive tests yielded negative results in one confirmed case. The patient was confirmed to be MERS-positive on the fifth test. These findings highlight the importance of implementing guidelines for laboratory testing, where patients should be retested by using a LRT specimen if initial testing is inconclusive or negative in a patient who is suspected to have MERS-CoV infection [1, 2].

In conclusion, URT specimens are not suitable for diagnosing MERS in early stages of MERS-CoV infection. Initial negative results should not rule out the possibility of MERS.

\section{Authors' Disclosures of Potential Conflicts of Interest}

No potential conflicts of interest relevant to this article were reported.

\section{REFERENCES}

1. Centers for Disease Control and Prevention. Novel coronavirus 2012 real-time RT-PCR assay. http://www.fda.gov/downloads/MedicalDevices/ Safety/EmergencySituations/UCM355572. pdf. (Updated on Jun 2014).

2. World Health Organization. Laboratory testing for Middle East Respiratory Syndrome Coronavirus. http://www.who.int/csr/disease/coronavirus_ infections/mers-laboratory-testing/en/ (Updated on Jun 2015).

3. Ki CS, Lee H, Sung H, Kim S, Seong MW, Yong D, et al. Korean Society for Laboratory Medicine Practice Guidelines for the Molecular Diagnosis of Middle East Respiratory Syndrome During an Outbreak in Korea in 2015. Ann Lab Med 2016;36:203-8.

4. Korea Ministry of Health and Welfare $(\mathrm{MoH})$ and Center for Disease Control and Prevention. Guidelins for MERS-CoV control. 3-3 ed. http://www. mers.go.kr/mers/html/jsp/main.jsp (Updated on Jun 2015).

5. Memish ZA, Al-Tawfiq JA, Makhdoom HQ, Assiri A, Alhakeem RF, Albarrak $A$, et al. Respiratory tract samples, viral load, and genome fraction yield in patients with Middle East respiratory syndrome. J Infect Dis 2014;210:1590-4

6. Assiri A, Al-Tawfiq JA, Al-Rabeeah AA, Al-Rabiah FA, Al-Hajjar S, Al-Barrak $A$, et al. Epidemiological, demographic, and clinical characteristics of 47 cases of Middle East respiratory syndrome coronavirus disease from Saudi Arabia: a descriptive study. Lancet Infect Dis 2013;13:75261.

7. Drosten C, Muth D, Corman VM, Hussain R, Al Masri M, HajOmar W, et al. An observational, laboratory-based study of outbreaks of middle East respiratory syndrome coronavirus in Jeddah and Riyadh, kingdom of Saudi Arabia, 2014. Clin Infect Dis 2015;60:369-77.

8. Kapoor M, Pringle K, Kumar A, Dearth S, Liu L, Lovchik J, et al. Clinical and laboratory findings of the first imported case of Middle East respiratory syndrome coronavirus to the United States. Clin Infect Dis 2014;59: 1511-8.

9. MERS Joint Public and Private Sector Response Committee (MERS Joint Committee). Laborator practice guidelines. http://www.mers.go.kr/mers/ html/jsp/main.jsp. (Updated on Jun 2015).

10. Centers for Disease Control and Prevention. Interim guidelines for collecting, handling, and testing clinical specimens from patients under investigation (PUIs) for Middle East Respiratory Syndrome Coronavirus (MERS-CoV). V2.1. http://www.cdc.gov/coronavirus/mers/guidelinesclinical-specimens.html (Updated on Jun 2015). 